\title{
Conditioning and latent inhibition with electrical stimulation of hippocampus
}

\author{
W. RONALD SALAFIA and ANDREA M. ALLAN \\ Fairfield University, Fairfield, Connecticut 06430
}

\begin{abstract}
This paper presents a review of recent research in which we have found disruption of conditioning of the rabbit nictitating membrane response with posttrial electrical stimulation of hippocampus. In addition, evidence is presented indicating that stimulation overlapping CS preexposure can facilitate or impede the development of latent inhibition. These data support the hypothesis that, in classical conditioning, the hippocampus acts as a modulator of sensory input.
\end{abstract}

For the past several years we have employed electrical stimulation to investigate the role of the limbic system and, in particular, the hippocampal formation in classical conditioning of the nictitating membrane response (NMR) of the rabbit. This paper begins with a brief review of our published work with posttrial stimulation (PTS), followed by the rationale for our current focus, namely, the effects of hippocampal stimulation on latent inhibition. Finally, the results of our initial latent inhibition experiments will be presented. A fundamental assumption underlying this research is that the hippocampus functions as a modulator of sensory input. Our goal is to understand the mechanism for modulation of a specific sensory input, the CS, as it affects conditioning.

\section{Conditioning with Posttrial \\ Hippocampal Stimulation}

Salafia, Romano, Tynan, Cloutman, Ahern, and Martino (Note 1) employed 5-sec bursts of posttrial stimulation (PTS) to hippocampus or overlying cortex with stimulation parameters set at values that would generate afterdischarges. Results indicated that hippocampal PTS presented immediately upon US offset severely disrupted conditioning of the NMR. More than twice as many trials were required for conditioning of the hippocampal group than for the conditioning of the unoperated and cortical-PTS control groups.

We could not be certain from this initial study that the effects were due solely to PTS, since prolonged primary and secondary afterdischarges frequently continued into the next trial. Therefore, Salafia, Romano, Tynan, and Host (1977) replicated and extended the study with fewer trials per session (18 CS-US paired trials and 2 CS-alone test trials) and a longer intertrial interval ( $2 \mathrm{~min})$, which prevented discharge activity from overlapping subsequent trials. Figure 1 presents the results of the first experiment of this study. A hippocampal PTS, a cortical PTS, and an unoperated control group were used, with stimulated

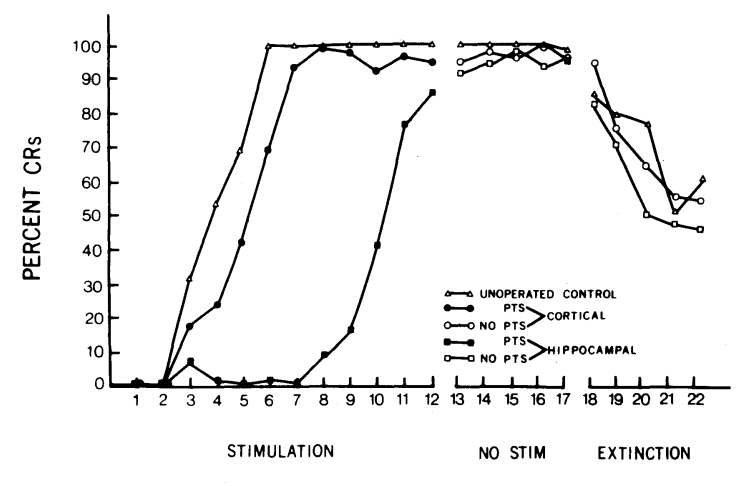

BLOCKS OF 20 TRIALS

Figure 1. Mean percent CRs as a function of trial blocks for the three groups under conditions of brain stimulation (for cortical and hippocampal groups), no stimulation, and extinction. (From Salafia et al., 1977.)

animals receiving a 5-sec burst of electrical stimulation immediately upon US-offset on paired trials. Hippocampal PTS again disrupted acquisition of conditioned responses. Although the cortically stimulated group conditioned slightly less rapidly than the unoperated control group, this difference did not approach statistical significance.

The second experiment involved two groups of hippocampally implanted animals (Figure 2 ). The experimental group was run for 12 days with PTS, while the control group was run for 8 days without stimulation, followed by 4 days with PTS. For the experimental group, there were 5 additional conditioning days without PTS, after which the stimulation was reintroduced for 2 days. It was found that the control group conditioned at about the same rate as the unoperated and cortical groups of Experiment 1, while the experimental group showed about the same level of disruption as had been seen in the hippocampal group of Experiment 1.

To summarize, the major effect of PTS appeared to be the postponement of CR emergence, with hip- 


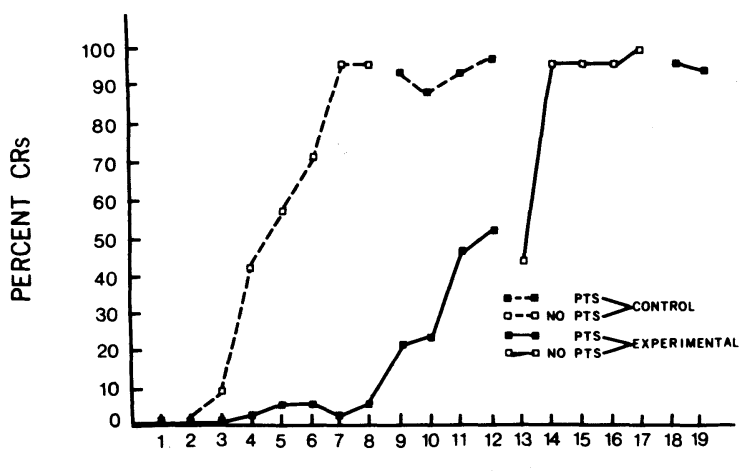

BLOCKS OF 20 TRIALS

Figure 2. Mean percent CRs as a function of trial blocks for the experimental and control groups, with and without brain stimulation. (From Salafia et al., 1977.)

pocampal animals taking about five sessions (100 trials) longer to condition. Once CRs began to occur, a comparison of slopes of the acquisition curves indicated that conditioning proceeded at about the same rate regardless of the presence or absence of stimulation. Hippocampal PTS initiated after conditioning had no effect on the elicitation of conditioned responses. Based on these results, we concluded that hippocampal PTS interfered with the establishment of the memory for the CS-US association but did not affect either registration of stimuli or the execution of conditioned or unconditioned responses.

Subsequent research has revealed that hippocampal PTS presented at intensities below seizure threshold produced about the same overall level of decrement in acquisition as did seizure-producing PTS (Salafia, Chiaia, \& Ramirez, 1979). This result suggested that the area stimulated, not brain seizures, is the critical element in the disruption effect.

One may not conclude definitively, on the basis of these results, that the hippocampal formation is the crucial structure in the disruption effects. First, it is possible that the effects were due to propagation of stimulation to other areas. Second, Kesner and Prokasy (Note 2) have had some difficulty recently in obtaining a similarly strong PTS effect. Although these investigators have employed different conditioning, stimulation, and delay parameters, their results, thus far at least, impose some limitation on the generality of the disruption effect. Finally, it is clear that the retardation of conditioning is not the result of the stimulation producing a "functional" lesion, since NMR acquisition has been shown to be unimpaired by bilateral lesions of hippocampus or septum (Lockhart \& Moore, 1975; Schmaltz \& Theios, 1972).

In spite of these concerns, substantial support for the hypothesis of hippocampal involvement in NMR conditioning has recently been reported. Berger, Alger, and Thompson (1976) found that hippocampal multipleunit discharges from CA1, CA3, and CA4 pyramidal cell layers and from the dentate granule cell layer formed a virtually exact temporal representation of the behavioral CR, but preceded it in time, typically by 25 to $35 \mathrm{msec}$. This unit activity was not found for spontaneous NMRs, nor was it found in the specifically unpaired control animals. More recently, Berger and Thompson (1977) found the same phaserelated hippocampal unit discharge as well as similar activity in the lateral, but not the medial, septal area. The hippocampus is further implicated in NMR conditioning by the recent discovery of a high correlation between the amount of hippocampal RSA recorded prior to conditioning and the subsequent rate of acquisition in the rabbit (Berry \& Thompson, 1978).

In summary, these electrophysiological studies as well as our PTS experiments seem to implicate the hippocampus in acquisition of the NMR. Contrary to these findings, hippocampal and septal lesions have not been found to retard acquisition. On the other hand, bilateral hippocampal lesions have been found to disrupt latent inhibition and blocking in the rabbit (Solomon, 1977; Solomon \& Moore, 1975). The latter results suggest a possible role for the hippocampus in attentional processes related to conditioning but perhaps not directly in the acquisition process. Reasoning that the attentional hypothesis may be more general, and able to encompass our PTS results, we have recently directed our attention toward the effects of electrical stimulation of hippocampus and cortex on the development and expression of latent inhibition.

\section{Hippocampus and Latent Inhibition}

Contemporary theories of inhibition in classical conditioning (e.g., Wagner \& Rescorla, 1972) distinguish two major inhibitory processes, viz, conditioned inhibition (CI) and latent inhibition (LI). Pavlovian CI involves the nonreinforced compounding of a CSwith an already excitatory $\mathrm{CS}+$. The organism must attend actively to the conditioned inhibitor in order to withhold responding to it or to a compound containing it.

Latent inhibition (LI), on the other hand, refers to retardation of subsequent conditioning by preexposure of the CS-to-be. A latent inhibitor compounded with an excitatory stimulus does not produce a deficit in responding, as does a conditioned inhibitor, suggesting that different mechanisms underlie the development of the two forms of inhibition.

Attempts to explain LI in behavioral terms have taken many forms, most of which have likened it to some type of habituation. As Mackintosh (1974) pointed out, the procedures which produce LI are operationally identical to those that produce habituation, the difference being that, with LI, the effects are measured in a subsequent learning task, while with habituation, responses are measured during stimulus exposure. However, there appears to be a growing consensus that the mechanisms underlying $\mathrm{LI}$ are more 
complex than habituation, although basically attentional in nature (Crowell \& Anderson, 1972; Siegel, 1970; Solomon, Brennan, \& Moore, 1974). Mackintosh (1973, 1975) and Weiss and Brown (1974) have suggested that, with preexposure, animals learn that the CS and US are uncorrelated; that is, since the CS signals no change in the probability of reinforcement, they learn to ignore it.

Although theories of hippocampal functioning have been many and varied (e.g., Douglas, 1967, 1972; Kimble, 1968; McCleary, 1966; O'Keefe \& Nadel, 1978 ), one notion that has dominated the theorizing for many years is that it plays a major role in behavioral inhibition. Douglas (1972) argued that the hippocampus might regulate attentional processes through modulation of midbrain arousal systems. In essence, he proposed that the hippocampus performs its inhibitory function primarily by acting as a nonreinforcement register, that is, it is responsible for determining that a stimulus has been received and is not followed by reinforcement, in which case it tunes out the stimulus, probably through modulation of the reticular core.

Douglas' theory appears to suggest a realistic physiological substrate for the prevalent behavioral explanation of LI, viz, learning to ignore. For instance, Ackil, Mellgren, Halgren, and Frommer (1969) found that hippocampectomized rats, in comparison with cortical and unoperated controls, failed to show LI in an active avoidance task. Solomon and Moore (1975) reported that while 450 preexposures of a tone that later became the CS in an NMR conditioning experiment resulted in the usual decrement in acquisition for normal and cortical control animals, rabbits with dorsal hippocampal lesions showed not only no deficit, but even a slight increment in acquisition rate. Solomon (1977) found that dorsal hippocampal ablation eliminated blocking of a light stimulus in Kamin's (1969) two-stage blocking paradigm, but did not affect the formation of Pavlovian CI. These results were taken as further support for Douglas' theory in that the blocking paradigm is assumed to involve the same sort of tuning-out process as LI, while, as indicated previously, CI involves active attention to the inhibitory CS rather than learning to ignore it. Recently, McFarland, Kostas, and Drew (1978) found that hippocampal lesions eliminated LI in taste aversion learning in rats, while Weiss, Friedman, and McGregor (1974) found that septal lesions abolished the LI effect in an active avoidance task in rats.

To summarize, the foregoing studies provide evidence that in tasks where normal animals learn to ignore redundant or otherwise irrelevant stimuli, animals with lesions in hippocampus and related limbic structures display a deficit in this sort of learning. In addition, Best and Best (1976) have shown that hippocampal unit activity during conditioning is altered by 15 to 20 nonreinforced preexposures to a tone, thus providing electrophysiological support for the hypothesis of hippocampal involvement in learning to ignore.

Schmaltz and Theois (1972) found that hippocampal lesioned rabbits displayed significantly faster acquisition of the classically conditioned NMR than normal or cortical control animals. However, dorsal hippocampectomy disrupted retention of extinction. Although Solomon (1977) suggested that the more rapid acquisition displayed by hippocampal animals may have resulted from an overreaction to a very salient CS and a very intense US, the difference could have been caused by a small amount of LI in the control animals. All animals had been subjected to six US-alone and six CS-alone presentations immediately prior to conditioning in order to test for the presence of URs to the CS in hippocampal animals. Although six trials are usually insufficient to produce measurable LI (Lubow, 1973), the LI effect can be enhanced by uncorrelated preexposures of the US along with the CS (Gamzu \& Williams, 1971; Kremer, 1971; Mackintosh, 1973). Alternatively (or additionally), it is possible that the early stages of conditioning may normally involve an initial tendency to ignore the CS, which is overcome with continued presentations of the CS-US pair. According to the present theorizing, then, the hippocampectomized animals should show somewhat less of this tendency. The presence of a deficit in retention of extinction for the hippocampal rabbits in the Schmaltz and Theios study strongly suggests a specific inhibitory deficit. Since the evidence presented earlier seems to favor hippocampal involvement in learning to ignore, these data imply that as extinction progresses, it may involve, at least partially, the development of learned inattention to the CS.

Moore (1979) has provided a neuronal model for NMR conditioning which may be able to integrate much of the research presented here. In essence, he suggests that, for the NMR, the actual site of associative convergence is within the brainstem reticular formation, while an "efference copy" of the CR could be rapidly projected to the septohippocampal complex. If the CS is redundant or otherwise irrelevant, tuning-out might occur under control of the septohippocampal complex via inhibitory projections back to reticular formation neurons. According to Moore's model, the phase-lead hippocampal and lateral septal response of Berger et al. (1976) and Berger and Thompson (1977) could be a neuronal representation that the CS has been recognized as relevant and is being tuned in, or at least is not being tuned out. This interpretation appears to be consistent with the results of Best and Best (1976), who found enhanced hippocampal unit activity during conditioning in cells from their nonpreexposed group, but little change in the preexposed group.

Similarly, it is not unreasonable to suppose that the hippocampal PTS effects may be associated with 
a tuning out process. Hippocampal PTS may either delay the process of establishing the relevance of the CS or induce a "tune-out" message for the CS in spite of the presence of a significant event, the US.

Given these considerations, we have recently begun a series of experiments to determine the effects of electrical stimulation of hippocampus on tasks involving learned inattention. It is assumed that this research will help to clarify the mechanism for the PTS disruption effects as well as the general mechanism for hippocampal involvement in the modulation of sensory input. Two experiments have been completed (Salafia \& Allan, in press, Note 3), and, although more research is needed, the results thus far have been encouraging and lend additional support to the hypothesis of hippocampal involvement in these processes.

\section{Hippocampal Stimulation and Latent Inhibition}

The first experiment (Salafia \& Allan, in press) was designed to determine if hippocampal stimulation (HS) at a fairly high intensity would attenuate LI when presented overlapping the CS during either preexposure or NMR conditioning. The basic design (Table 1) involved five groups of rabbits. Group 1 sat in the experimental enclosure for an amount of time equivalent to that of the preexposed groups and then received 50 CS-US paired trials, interspersed with 4 CS-alone test trials, per day for 7 days. Group 2 received 150 preexposures of the tone CS followed by the same sequence of conditioning trials. Groups 3 and 5 were preexposed and conditioned as Group 2 was, except that, for Group 3, HS overlapped the CS during preexposure, while, for Group 5, HS overlapped the CS during conditioning. Finally, Group 4 was the same as Group 1, except that HS overlapped the CS during conditioning.

Hippocampal stimulation consisted of $800-\mathrm{msec}$ trains of 2-mesc pulses at a rate of 100 pps and an intensity of $50 \mu \mathrm{A}$. For both preexposure and conditioning, the CS was a $1,000-\mathrm{Hz}, 90-\mathrm{dB}$ tone presented at 30 -sec intervals for 800 msec against a 65 -dB background noise. In the conditioning phase, the US was a $2-\mathrm{mA}, 60-\mathrm{Hz}$ shock delivered to circumorbitally attached wound clips and overlapping the last $50 \mathrm{msec}$ of CS presentation.

Detailed surgical, conditioning, and histological procedures have been presented elsewhere (Salafia et al., 1979; Salafia \& Allan, in press). Operated animals received pedestals containing four stainless steel insect pins (size 00), insulated but for the last $.5 \mathrm{~mm}$. After recovery and preparation, the animals were placed in cubicles and run four at a time under the conditions specified above.

The basic results, also depicted in Table 1, indicate that the SIT-control animals (Group 1) showed the most rapid and stable acquisition, while the animals in Group 2 made a mean of 115 fewer CRs, indicating significant retardation of conditioning with CS preexposure. Significant attenuation of LI was evi-
Table 1

Basic Design of Experiment 1 and Results Expressed in Terms of the Mean Total Number of CRs Made During Conditioning

\begin{tabular}{cllc}
\hline Group & Preexposure & Conditioning & $\begin{array}{c}\text { Mean Total } \\
\text { CRs }\end{array}$ \\
\hline 1 & SIT & CS-US & 250 \\
2 & CS & CS-US & 135 \\
3 & CS/HS & CS-US & 174 \\
4 & SIT & CS/HS-US & 228 \\
5 & CS & CS/HS-US & 198 \\
\hline
\end{tabular}

dent in both Group 3, where HS overlapped CS preexposure, and Group 5, where HS overlapped the CS during conditioning, after preexposure. Thus, the first major question was answered in the affirmative; hippocampal stimulation attenuates LI in the rabbit, although it does not eliminate LI as seems to be the case with hippocampal lesions (Solomon \& Moore, 1975).

The performance of Group 4, where HS overlapped conditioning in the absence of preexposure, was only slightly, but not significantly, poorer than that of Group 1. This result addresses one of the major questions which provided the impetus for the LI experiments, namely the mechanism for hippocampal PTS effects (Salafia et al., 1977, 1979, Note 1). It had been suggested that the decrements in conditioning observed by Salafia et al. were due to direct interference with the establishment of memory. Alternatively, Moore (1979) suggested that PTS might affect conditioning by way of attentional mechanisms. The present results provide indirect support for the latter alternative. It seems unlikely, if HS overlapping CS-US presentations has virtually no effect on conditioning, that hippocampal PTS would have a direct effect. This conclusion must be tempered, however, by the fact that, in the PTS studies, stimulation was presented for $5 \mathrm{sec}$, more than six times the train duration of the present experiment. Such a long train of pulses presented after each trial could have effects different from those of a short train overlapping CS-US presentation.

The second experiment (Salafia \& Allan, Note 3) was designed to assess the effects of lower level HS on LI. Before conducting the experiment, two intervening studies established that no difference in conditioning or LI effects could be found as a function of the surgical procedures and that 300 preexposures produced a less variable LI effect than 150 preexposures. Thus, our second LI experiment involved substantive differences in preexposure and conditioning parameters in addition to the changes in stimulation parameters. Additionally, we decided to focus attention on stimulation during preexposure for this experiment.

The design of Experiment 2 involved four groups, as designated in Table 2. The SIT and PRE groups were unoperated. Group SIT was placed in the cubicles for an amount of time corresponding to that of the preexposed groups, then conditioned for 3 days with 
Table 2

Basic Design of Experiment 2 and Results Expressed in Terms of the Mean Total Number of CRs Made During Conditioning

\begin{tabular}{ccc}
\hline Group & $\begin{array}{c}\text { Number of } \\
\text { Preexposures }\end{array}$ & $\begin{array}{c}\text { Mean Total } \\
\text { CRs }\end{array}$ \\
\hline SIT & 0 & 263 \\
PRE & 300 & 191 \\
HS & 300 & 152 \\
COS & 300 & 175 \\
\hline
\end{tabular}

100 CS-US trials per day interspersed with 3 CS-alone test trials. Group PRE received 300 preexposures followed by the same sequence of conditioning trials. Groups HS and COS were run under the same conditions as Group PRE, except that during preexposure the onset of each tone was accompanied by the onset of hippocampal (HS) or cortical (COS) stimulation. For preexposure and conditioning, the CS was a $1,000-\mathrm{Hz}, 90-\mathrm{dB}$ tone, presented at 30-sec intervals for $300 \mathrm{msec}$. Again, the US was a $2-\mathrm{mA}, 60-\mathrm{Hz}$ shock overlapping the last $50 \mathrm{msec}$ of CS presentation. Hippocampal and cortical stimulation consisted of 600 -msec trains of $200-\mu \mathrm{sec}$ pulses at a rate of $40 \mathrm{pps}$ and an intensity of $30 \mu \mathrm{A}$.

The conditioning results, also presented in Table 2 , indicated that again there was a large LI effect. Although, in terms of the difference between Groups SIT and PRE, the effect was not as large as that found in Experiment 1, it was substantially more stable and reliable. Thus, the changes in preexposure and conditioning parameters achieved the desired effect. Interestingly, both stimulated groups displayed significant augmentation of LI, with Group HS displaying the most. Clearly, the basic result of lowering the stimulation level (in terms of amplitude, pulse rate, pulse width) was an effect opposite to that found in Experiment 1 with higher intensity stimulation.

Substantial variability in the performance of stimulated animals had been noted in previous PTS experiments (Salafia et al., 1977, 1979, Note 1) as well as in the present experiments. Previously there was no obvious pattern to this variability. In Experiment 2, however, it was observed that three of the animals in Group HS showed no apparent effect of the stimulation, while the remaining four showed extreme retardation of conditioning. In an attempt to find an explanation for the apparent discrepancy, a test for stimulation effects was performed after the preexposure and conditioning phase had been completed. Initially, the test involved identifying stimulation parameters that had some noticeable effect on behavioral CRs. Next, animals were given an extinction series in which some trials were presented with the CS alone, while in others, brain stimulation overlapped the CS. During the test, some animals from Group PRE were also run for comparison.

The most important aspects of the test for stimulation effects are depicted in Table 3. Although Group PRE was unoperated and therefore received no
Table 3

Mean Percentage of CRs Made During the Specified Trials in the Test for Stimulation Effects

\begin{tabular}{crcc} 
Group & CS-US & CS/S & CS Alone \\
\hline PRE & 100 & 61 & 49 \\
COS & 92 & 38 & 63 \\
HS-1 & 98 & 0 & 78 \\
HS-2 & 100 & 93 & 81 \\
\hline
\end{tabular}

stimulation, the performance of this group during the periods when the other groups received stimulation is listed under the CS/S column. Additionally, it should be noted that, since more CS/S trials occurred earlier in the test and more CS-alone trials later, the difference in the performance of Group PRE during the two periods does not imply an unusual effect.

During the CS-US trials, which were run to establish a baseline, all groups performed at approximately the same high level. In general, the animals in Group COS showed somewhat depressed responding in the CS/S period, suggesting a modest amount of CR suppression due to the stimulation. On the other hand, animals in the HS group performed so differently that the group was reclassified into two subgroups, designated HS-1 and HS-2.

Not one animal in Group HS-1 made a CR during the CS/S periods. Although URs were not affected in any discernible way, this group showed complete suppression of CRs. On the other hand, Group HS-2 showed strong evidence of enhancement of responding, in that these animals responded at the highest rate of all on CS/S trials. For both of these subgroups, however, performance in the CS-alone periods was at a high level and revealed that relatively little extinction had taken place.

These unexpected results suggest that the same level of hippocampal stimulation is capable of producing quite different behavioral and physiological effects. The basis for the different effects is not clear. The obvious explanation of different or variable electrode placements was discounted because of the precision and consistency noted in the histologies. It is possible that differential effects of the same level of stimulation were the result of an interaction between the stimulation and different levels of ongoing hippocampal activity. In support of this argument is a recent study (Berry \& Thompson, 1978) in which a high correlation was found between conditioning rate and hippocampal RSA generated prior to conditioning.

Given the results of the test for stimulation effects, we returned to the prior conditioning performance for some further analysis and found that the animals in Group HS-1 had conditioned slightly, but not significantly, faster ( $\bar{X}=201 \mathrm{CRs})$ than Group PRE $(\bar{X}=191 \mathrm{CRs})$. On the other hand, animals in Group HS-2 had shown substantially more retardation of conditioning ( $\overline{\mathrm{X}}=111 \mathrm{CRs})$ than Group PRE. Thus, it seems virtually certain that much of the 
variability observed in stimulated groups is not random, but rather that it is due to differential stimulation effects.

One final aspect of Experiment 2 which has not previously been discussed is the performance of Group COS. Recall that this group also showed greater retardation of conditioning than Group PRE, but not as much as Group HS (Table 2). Furthermore, some animals in Group COS had shown evidence of CR suppression during the test but, again, not as much as those in Group HS-1. Moreover, during one phase of Experiment 1, we had run three cortically stimulated groups (not heretofore mentioned) under the same preexposure and conditioning parameters as the hippocampally stimulated groups (Groups 3-5). The animals in these groups also displayed behavior in the same direction as that of their hippocampal counterparts, but again the effects were small and not statistically reliable. In general, then, the effects of COS seem to be similar to those of HS, but much smaller.

These COS effects could be taken as evidence that the effect of stimulation, such as that employed in these experiments, is very general and therefore cannot be ascribed to the functioning of any specific system, such as the hippocampal complex. However, recent neuroanatomical evidence (see, e.g., Swanson, 1978 , for a review) indicates that cortical input to hippocampus is much more massive than heretofore suspected. Therefore, although conclusive evidence must await further research, it seems reasonable to suggest that the modest COS effects were simply less direct HS effects.

\section{General Discussion and Conclusions}

The results of the two experiments summarized here indicate, first, that intense hippocampal stimulation attenuates LI, while lower level stimulation augments it. Second, both attenuation and augmentation effects were variable, but the variability is not random. Rather, it seems to be due to a differential stimulation effect, which itself may result from an interaction between different levels of ongoing hippocampal activity and brain stimulation. Third, although cortical stimulation seems to affect LI in a manner similar to that of hippocampal stimulation, the effects are small and, in most cases, not statistically reliable. These may still be hippocampal effects, however, in light of the neuroanatomical evidence for massive input to hippocampus from various cortical areas. Finally, hippocampal stimulation overlapping CS-US presentation in the absence of preexposure appears to have little effect on conditioning. This suggests, indirectly, that the decrement in conditioning found in our previous posttrial hippocampal stimulation studies may have been due to the effects of PTS on attentional processes rather than on conditioning.

In general, the results of the LI studies (Salafia \& Allan, in press, Note 3) summarized here, as well as those of the PTS experiments (Salafia et al., 1977, 1979, Note 1), appear to be congruent with the view that, in classical conditioning, a major function of hippocampus is the modulation of sensory input. More specifically, the data add support to a neuronal model for NMR conditioning such as that proposed by Moore (1979). It is suggested that hippocampal stimulation may produce changes in the neural representation of the CS in hippocampus. These changes could then affect the character of the neural activity projected from hippocampus to associative centers. From a behavioral point of view, the effects of such changes would be alterations in the capacity of the stimulated animal to learn to ignore irrelevant stimuli. These changes would then be reflected in the enhanced and retarded conditioning that we have observed. We are continuing this line of research with the expectation that it will answer some of the questions either raised or left unanswered by the available data.

\section{REFERENCE NOTES}

1. Salafia, W. R., Romano, A. G., Tynan, T., Cloutman, K., Ahern, K., \& Martino, L. J. Posttrial hippocampal stimulation and conditioning of the rabbit (Oryctolagus cuniculus) nictitating membrane response. Paper presented at the meeting of the Psychonomic Society, Boston, November 1974.

2. Kesner, R. P., \& Prokasy, W. F. Personal communication, 1979.

3. Salafia, W. R., \& Allan, A. M. Augmentation of latent inhibition by electrical stimulation of hippocampus during preexposure. Manuscripts submitted for publication, 1979.

\section{REFERENCES}

Ackil, J. E., Mellgren, R. L., Halgren, C., \& Frommer, G. P. Effects of CS preexposure on avoidance learning in rats with hippocampal lesions. Journal of Comparative and Physiological Psychology, 1969, 69, 739-747.

Berger, T. W., Alger, B., \& Thompson, R. F. Neuronal substrate of classical conditioning in the hippocampus. Science, 1976, 192, 483-485.

Berger, T. W., \& Thompson, R. F. Limbic system interrelations: Functional division among hippocampal-septal connections. Science, 1977, 197, 587-589.

Berry, S. D., \& Thompson, R. F. Prediction of learning rate from the hippocampal electroencephalogram. Science, 1978, 200, 1298-1300.

Best, M. R., \& Best, P. J. The effects of state of consciousness and latent inhibition on hippocampal unit activity in the rat during conditioning. Experimental Neurology, 1976, 51, 564-573.

Crowell, C. R., \& Anderson, D. C. Variations in intensity, interstimulus interval, and interval between preconditioning CS exposures and conditioning with rats. Journal of Comparative and Physiological Psychology, 1972, 79, 291-298.

Douglas, R. J. The hippocampus and behavior. Psychological Bulletin, 1967, 67, 416-442.

Douglas, R. J. Pavlovian conditioning and the brain. In R. A. Boakes \& M. S. Halliday (Eds.), Inhibition and learning. New York: Academic Press, 1972.

GamzU, E., \& Williams, D. R. Classical conditioning of a complex skeletal response. Science, 1971, 171, 923-925.

Kamin, L. J. Predictability, surprise, attention, and conditioning. In B. Campbell \& R. Church (Eds.), Punishment and aversive behavior. New York: Appleton-Century-Crofts, 1969. 
Kimble, D. P. Hippocampus and internal inhibition. Psychological Bulletin, 1968, 70, 285-295.

KREMER, E. F. Truly random and traditional control procedures in CER conditioning in the rat. Journal of Comparative and Physiological Psychology, 1971, 76, 441-448.

Lockhart, M., \& Moore, J. W. Classical differential and operant conditioning in rabbits with septal lesions. Journal of Comparative and Physiological Psychology, 1975, 88, 147-154.

LuBow, R. E. Latent inhibition. Psychological Bulletin, 1973, 79, 398-407.

Mackintosh, N. J. Stimulus selection: Learning to ignore stimuli that predict no change in reinforcement. In R. A. Hinde \& J. Stevenson-Hinde (Eds.), Constraints on learning. London: Academic Press, 1973.

MaCKIntosh, N. J. The psychology of animal learning. London: Academic Press, 1974.

Mackintosh, N. J. A theory of attention: Variations in the associability of stimuli with reinforcement. Psychological Review, 1975, 82, 276-298.

MCCleARY, R. A. Response-modulating functions of limbic system: Initiation and suppression. In E. Stellar \& J. M. Sprague (Eds.), Progress in physiological psychology (Vol. 1). New York: Academic Press, 1966.

McFarland, D. J., Kostas, J., \& Drew, W. G. Dorsal hippocampal lesions: Effects of preconditioning CS exposure on flavor aversion. Behavioral Biology, 1978, 22, 398-404.

Moore, J. W. Brain processes and conditioning. In A. Dickinson \& R. A. Boakes (Eds.), Associative mechanisms in conditioning. A memorial volume for Jerzy Konorski. Hillsdale, N.J: Erlbaum, 1979.

O'KeEFE, J., \& NADEL, L. The hippocampus as a cognitive map. Oxford: Oxford University Press, 1978.

Salafia, W. R., \& Allan, A. M. Attenuation of latent inhibition by electrical stimulation of hippocampus. Physiology \& Behavior, in press.

Salafia, W. R., Chiaia, N. L., \& Ramirez, J. J. Retardation of rabbit nictitating membrane conditioning by subseizure electrical stimulation of hippocampus. Physiology \& Behavior, 1979, 22, 451-455.
Salafia, W. R., Romano, A. G., Tynan, T., \& Host, K. C. Disruption of rabbit nictitating membrane conditioning by posttrial electrical stimulation of hippocampus. Physiology \& Behavior, 1977, 18, 207-212.

Schmaltz, L. W., \& Theios, J. Acquisition and extinction of a classically conditioned response in hippocampectomized rabbits. Journal of Comparative and Physiological Psychology, 1972, 79, 328-333.

Siegel, S. Retention of latent inhibition. Psychonomic Science, 1970, 20, 161-162.

Solomon, P. R. Role of the hippocampus in blocking and conditioned inhibition of the rabbit's nictitating membrane response. Journal of Comparative and Physiological Psychology, 1977, 91, 407-417.

Solomon, P. R., Brennan, G., \& Moore, J. W. Latent inhibition of the rabbit's nictitating membrane response as a function of CS intensity. Bulletin of the Psychonomic Society, 1974, 4, 445-448.

Solomon, P. R., \& Moore, J. W. Latent inhibition and stimulus generalization of the classically conditioned nictitating membrane response in rabbits following dorsal hippocampal ablation. Journal of Comparative and Physiological Psychology, 1975, 89, 1192-1203.

Swanson, L. W. The anatomical organization of septo-hippocampal projections. In Ciba Foundation Symposium 58 (new series), Functions of the septo-hippocampal system. New York: Elsevier, 1978.

Wagner, A. R., \& Rescorla, R. A. Inhibition in Pavlovian conditioning: Application of a theory. In R. A. Boakes \& M. S. Halliday (Eds.), Inhibition and learning. New York: Academic Press, 1972.

Weiss, K. R., \& Brown, B. L. Latent inhibition: A review and a new hypothesis. Acta Neurobiologica Experimentalis, 1974, 34, 301-316.

Weiss, K. R., Friedman, R., \& McGregor, S. Effects of septal lesions on latent inhibition and habituation of the orienting responses in rats. Acta Neurobiologica Experimentalis, 1974, 34, 491-504. 